\title{
On Parametric Stability Margin Maximization Using State Feedback Pole Assignment
}

\author{
Tingshu $\mathrm{Hu}$ \\ Department of Electrical and Computer Engineering \\ University of Waterloo \\ Waterloo, Ontario \\ CANADA N2L 3G1
}

\author{
James $\operatorname{Lam}^{\dagger}$ \\ Department of Mechanical Engineering \\ University of Hong Kong \\ Pokfulam Road \\ HONG KONG
}

\begin{abstract}
In this paper, the maximization of the parametric stability margin of state-space uncertain systems under the constraints of pole assignment is investigated. The class of systems considered is where the uncertainty may be modelled as the, possibly nonlinear, variation of a parameter appearing in the entries of the system and input matrices. The continuity and differentiability of the stability margin are discussed. A gradient-based procedure is formulated for the maximization of the stability margin with the corresponding gradient provided. Numerical examples are used to illustrate the technique.
\end{abstract}

Keywords: stability margin, pole assignment.

\section{Introduction}

One way to represent uncertainties or perturbations in control systems is to model them as parameters in the system descriptions. From a control-theoretic point of view, the influence of these parameters on the stability of control systems has naturally become the major issue for consideration in parametric uncertain systems. Stability robustness condition was given by Patel and Toda [7] for perturbation in the state matrix without exploiting any structural information of the perturbation. Subsequently, Yedavalli [13], Zhou and Khargonekar [14] gave improved results for structured perturbation. These and many other results assumed the perturbation in the system matrix as affine functions of the parameters. Moreover, the results presented are focussed on providing bounds on the parameters to ensure stability rather than for control synthesis. The behaviour of systems under perturbations modelled as polynomial functions of an uncertain parameter vector is considered by Tesi and Vicino $[11,12]$. Other numerical approaches related to certain robust stability margins computation may be found in $[5,8,10]$. The formulations are based on specific structures in the way which the parameters appeared in the characteristic polynomial coefficients. While these approaches lead to nice theoretical results

\footnotetext{
This work is supported by HKU CRCG 337/064/0028
}

and taylor-made computation algorithms, the general nonlinear case has not been fully addressed.

In control system design, it would be important to construct a closed-loop system such that it is maximally tolerable towards uncertainties. For a completely state controllable realization, it is well known that the closed-loop poles may be assigned, via constant state feedback, to any set of self-conjugate complex numbers of cardinality equal to the statedimension. For multi-input systems, the nonuniqueness of the feedback gain to achieve a given pole assignment specification may be exploited to optimize a variety of system performance indices. The most common application of this idea is robust pole assignment (see $[1,4,6]$ and references therein). There is little work on utilizing the freedom in the state feedback gain matrices to improve stability margin. The obvious reason is that pole assignment itself imposes constraints to the feedback systems and inevitably reduces the overall achievable stability margin if only closed-loop stability is imposed. However, the tradeoff between pole assignment constraints and optimum performance is often justifiable in view of implementation since optimal solutions may have undesirable transient behavior or unacceptably large gain.

Motivated by the above reasons, this paper considers the maximization of a parametric stability margin under the constraints of pole assignment via state feedback. As a first step towards a more general computation procedure, it is assumed that the (nonlinear) perturbation is parametrized by a single parameter.

\section{Stability Margin}

Consider the following parametric uncertain system

$$
\dot{x}=M(F, p) x
$$

where $x \in \mathbf{R}^{n}$ is the state, $F$ is a real matrix containing all the design parameters, $p \in \mathbf{R}$ is the uncertain parameter and $M(F, p) \in \mathbf{R}^{n \times n}$ is a continuously differentiable matrix function in $F$ and $p$. 
For a given $F$, suppose $M(F, 0)$ is stable, then there exists a real number $r>0$ such that $M(F, p)$ is stable for $p \in(-r, r)$. A practical problem is to select an $F$ such that this $r$ is maximized. The stability margin is characterized as follows.

Definition 1 Let $\mathcal{F}$ be the set of $F$ such that $M(F, 0)$ is stable. For $F \in \mathcal{F}$, define

$$
\rho_{M}(F):=\left\{\begin{array}{l}
\min \{|p|: M(F, p) \text { is unstable }\} \\
\infty, \quad \text { if } M(F, p) \text { is stable for } p \in \mathbf{R}
\end{array}\right.
$$

In this paper, we are particularly interested in the following closed-loop system,

$$
\dot{x}=M(F, p) x=[A(p)+B(p) F] x
$$

where $F \in \mathbf{R}^{m \times n}$ is the state feedback matrix. And $A(p) \in \mathbf{R}^{n \times n}, B(p) \in \mathbf{R}^{n \times m}$ are matrix functions that are continuously differentiable with respect to the uncertain parameter $p \in \mathbf{R}$. In this paper, we are interested in selecting an $F$ such that $\rho_{M}(F)$ is maximized under the constraint of pole assignment. For simplicity, denote $A(0)=A_{0}, B(0)=B_{0}$.

Stability Margin Maximization Under Pole Assignment Problem: Suppose $A(p), B(p)$ are differentiable matrix functions of $p$. The problem is to find a state feedback $F$ such that $\rho_{M}(F)$ is maximized under the constraint that $A_{0}+B_{0} F$ has the prescribed eigenvalues.

Since $p$ is a scalar, for a given $F, \rho_{M}(F)$ can be computed by the bisection method. It is clear that functions of this kind are very complicated and can possess many discontinuities. To maximize $\rho_{M}(F)$ based on gradient information, one must have knowledge about under what condition $\rho_{M}(F)$ is continuous and differentiable.

\subsection{Continuity and Differentiability of Stabil- ity Margin}

To maintain the generality of the development, let $M(F, p) \in \mathbf{R}^{n \times n}$ be a continuously differentiable matrix function of $F \in \mathbf{R}^{m \times n}$ and $p \in \mathbf{R}$. Assume that all the eigenvalues of $M(F, 0)$ have negative real parts. Denote

$$
Q(F, p)=\left[\begin{array}{cc}
M(F, p) & 0 \\
0 & M(F,-p)
\end{array}\right],
$$

then

$\rho_{M}(F)=\left\{\begin{array}{l}\min \left\{p>0: \operatorname{Re} \lambda_{i}[Q(F, p)]=0 \text { for some } i\right\} \\ \infty, \quad \text { if } \operatorname{Re} \lambda_{i}[Q(F, p)] \neq 0 \forall p>0 \text { and } i .\end{array}\right.$ where $\operatorname{Re} \lambda_{i}[\cdot]$ denotes the real part of the $i$ th eigenvalue of a matrix. For a given $F$, the eigenloci of $Q(F, p)$ can be drawn as $p$ varies continuously. Each locus $\lambda_{i}[Q(F, p)]$ is continuous and piecewise smooth. Then $\rho_{M}(F)$ equals to the smallest $p$ at which one of the loci hits the imaginary axis.

Theorem 1 For a given $F_{0}$, let $p_{0}=\rho_{M}\left(F_{0}\right)$. Suppose $Q\left(F_{0}, p_{0}\right)$ has $\ell$ distinct eigenvalues $\lambda_{i}\left[Q\left(F_{0}, p_{0}\right)\right]$, $1 \leq i \leq \ell$ on the imaginary axis, then

1. $\rho_{M}(F)$ is continuous in a neighborhood of $F_{0}$ if there is one $i, 1 \leq i \leq \ell$ such that

$$
\frac{\partial \operatorname{Re} \lambda_{i}\left[Q\left(F_{0}, p_{0}\right)\right]}{\partial p} \neq 0
$$

2. $\rho_{M}(F)$ is differentiable at $F_{0}$ if

$$
\frac{\partial \operatorname{Re} \lambda_{i}\left[Q\left(F_{0}, p_{0}\right)\right]}{\partial p} \neq 0 \text { for } 1 \leq i \leq \ell
$$

and the following $\ell$ items are equal

$$
\frac{\frac{\partial \operatorname{Re} \lambda_{i}\left[Q\left(F_{0}, p_{0}\right)\right]}{\partial F}}{\frac{\partial \operatorname{Re} \lambda_{i}\left[Q\left(F_{0}, p_{0}\right)\right]}{\partial p}}, \quad 1 \leq i \leq \ell
$$

In this case, the partial derivative of $\rho_{M}(F)$ at $F_{0}$ is given as,

$$
\frac{\partial \rho_{M}\left(F_{0}\right)}{\partial F}=-\frac{\frac{\partial \operatorname{Re} \lambda_{1}\left[Q\left(F_{0}, p_{0}\right)\right]}{\partial F}}{\frac{\partial \operatorname{Re} \lambda_{1}\left[Q\left(F_{0}, p_{0}\right)\right]}{\partial p}}
$$

To prove the above theorem, define for $i=1,2, \ldots, 2 n$,

$$
\rho_{M}^{i}(F):=\left\{\begin{array}{l}
\min \left\{p>0: \operatorname{Re} \lambda_{i}[Q(F, p)]=0\right\} \\
\infty, \quad \text { if } \operatorname{Re} \lambda_{i}[Q(F, p)] \neq 0 \forall p>0 .
\end{array}\right.
$$

It is easy to see that

$$
\rho_{M}(F)=\min \left\{\rho_{M}^{i}(F), i=1,2, \ldots, 2 n\right\}
$$

For each $\rho_{M}^{i}(F)$, we have the following result.

Lemma 1 For a given $F_{0}$, assume $\rho_{M}^{i}\left(F_{0}\right)<\infty$. Let $p_{0}=\rho_{M}^{i}\left(F_{0}\right)$ (hence $\operatorname{Re} \lambda_{i}\left[Q\left(F_{0}, p_{0}\right)\right]=0$ ). Suppose that the following conditions are satisfied,

$$
\begin{aligned}
& \text { 1. } \lambda_{i}\left[Q\left(F_{0}, p_{0}\right)\right] \text { is a simple eigenvalue of } Q\left(F_{0}, p_{0}\right) \\
& \text { 2. } \frac{\partial \operatorname{Re} \lambda_{i}\left[Q\left(F_{0}, p_{0}\right)\right]}{\partial p} \neq 0 \text {. }
\end{aligned}
$$

then $\rho_{M}^{i}(F)$ is continuously differentiable in a neighborhood of $F_{0}$ with

$$
\frac{\partial \rho_{M}^{i}\left(F_{0}\right)}{\partial F}=-\frac{\frac{\partial \operatorname{Re} \lambda_{i}\left[Q\left(F_{0}, p_{0}\right)\right]}{\partial F}}{\frac{\partial \operatorname{Re} \lambda_{i}\left[Q\left(F_{0}, p_{0}\right)\right]}{\partial p}}
$$


Proof: The result then follows from an application of the implicit function theorem with (6).

Lemma $2 \rho_{M}^{i}(F)$ is semi-continuous from above. That is, given $F_{0}$, if $\rho_{M}^{i}\left(F_{0}\right)>\alpha$, then there exists $\delta>0$, such that $\rho_{M}^{i}(F)>\alpha$ whenever $\left\|F-F_{0}\right\|<\delta$.

Proof of Theorem 1: Notice that $\rho_{M}^{i}\left(F_{0}\right)=\rho_{M}\left(F_{0}\right)$ for $i \leq \ell$ and $\rho_{M}^{i}\left(F_{0}\right)>\rho_{M}\left(F_{0}\right)$ for $i>\ell$.

1. W.l.o.g., assume that $\frac{\partial \operatorname{Re} \lambda_{1}\left[Q\left(F_{0}, p_{0}\right)\right]}{\partial p} \neq 0$, it follows from Lemma 1 that $\rho_{M}^{1}(F)$ is continuous at $F_{0}$. Thus for any $\xi>0$, there exists $\delta_{1}>0$ such that when $\left\|F-F_{0}\right\|<\delta_{1},\left|\rho_{M}^{1}(F)-\rho_{M}^{1}\left(F_{0}\right)\right|<\xi$. Since $\rho_{M}^{i}\left(F_{0}\right)>\rho_{M}^{1}\left(F_{0}\right)-\xi$, by Lemma 2, there exists $\delta \in\left(0, \delta_{1}\right]$ such that when $\left\|F-F_{0}\right\|<\delta$, $\rho_{M}^{i}(F)>\rho_{M}^{1}\left(F_{0}\right)-\xi, i=2, \ldots, 2 n$. This implies that $\rho_{M}(F)>\rho_{M}\left(F_{0}\right)-\xi$. On the other hand, since $\rho_{M}^{1}(F)<\rho_{M}\left(F_{0}\right)+\xi$, we have $\rho_{M}(F)<\rho_{M}\left(F_{0}\right)+\xi$ by (6). It follows that $\left|\rho_{M}(F)-\rho_{M}\left(F_{0}\right)\right|<\xi$ for all $F$ satisfying $\left\|F-F_{0}\right\|<\delta$. This shows that $\rho_{M}(F)$ is continuous at $F_{0}$.

2. When the conditions are satisfied, $\rho_{M}^{i}(F), i \leq \ell$ are continuous at $F_{0}$. Let $\xi=\frac{1}{2} \min _{i>\ell}\left(\rho_{M}^{i}\left(F_{0}\right)-\right.$ $\left.\rho_{M}\left(F_{0}\right)\right)$, there exists $\delta>0$ such that when $\| F-$ $F_{0} \|<\delta,\left|\rho_{M}^{i}(F)-\rho_{M}\left(F_{0}\right)\right|<\xi$ for $i \leq \ell$ and $\rho_{M}^{i}(F)>\rho_{M}\left(F_{0}\right)+\xi$ for $i>\ell$ (by Lemma 2). This shows $\rho_{M}(F)=\min \left\{\rho_{M}^{i}(F), i=1,2, \ldots, \ell\right\}$ when $\left\|F-F_{0}\right\|<\delta$. Thus, together with the conditions, we have $\rho_{M}(F)$ continuous differentiable and the partial derivative formula (5) follows.

\subsection{Partial Derivative Formulas for Stability Margin}

Here, we provide formulas to compute $\frac{\partial \operatorname{Re} \lambda_{1}[Q(F, p)]}{\partial F}$ and $\frac{\partial \operatorname{Re} \lambda_{1}[Q(F, p)]}{\partial p}$. Denote the left eigenvector and the right eigenvector of $Q\left(F_{0}, p_{0}\right)$ corresponding to $\lambda_{1}$ as $t^{T}$ and $v, t^{T} v=1$. Furthermore, $t, v$ are partitioned as $t^{T}=\left[\begin{array}{cc}t_{1}^{T} & t_{2}^{T}\end{array}\right], v^{T}=\left[\begin{array}{cc}v_{1}^{T} & v_{2}^{T}\end{array}\right], t_{1}, t_{2}, v_{1}, v_{2} \in$ $\mathrm{C}^{n}$. We have

$$
\begin{aligned}
& \frac{\partial \operatorname{Re} \lambda_{1}\left[Q\left(F_{0}, p_{0}\right)\right]}{\partial p}=\operatorname{Re} \frac{\partial \lambda_{1}\left[Q\left(F_{0}, p_{0}\right)\right]}{\partial p} \\
& \frac{\partial \operatorname{Re} \lambda_{1}\left[Q\left(F_{0}, p_{0}\right)\right]}{\partial f_{j k}}=\operatorname{Re} \frac{\partial \lambda_{1}\left[Q\left(F_{0}, p_{0}\right)\right]}{\partial f_{j k}}
\end{aligned}
$$

where $F=\left[f_{j k}\right]$. In particular, when $M(F, p)=$ $A(p)+B(p) F$, then it follows that

$$
\frac{\partial \operatorname{Re} \lambda_{1}\left[Q\left(F_{0}, p_{0}\right)\right]}{\partial F}=\left[\operatorname{Re}\left(v_{1} t_{1}^{T}\right) B\left(p_{0}\right)+\operatorname{Re}\left(v_{2} t_{2}^{T}\right) B\left(-p_{0}\right)\right]^{T}
$$

and consequently, we have

$$
\begin{aligned}
& \frac{\partial \rho_{M}\left(F_{0}\right)}{\partial F}= \\
& \frac{-\left[\operatorname{Re}\left(v_{1} t_{1}^{T}\right) B\left(p_{0}\right)+\operatorname{Re}\left(v_{2} t_{2}^{T}\right) B\left(-p_{0}\right)\right]^{T}}{\operatorname{Re}\left\{t_{1}^{T}\left[A^{\prime}\left(p_{0}\right)+B^{\prime}\left(p_{0}\right) F\right] v_{1}-t_{2}^{T}\left[A^{\prime}\left(-p_{0}\right)+B^{\prime}\left(-p_{0}\right) F\right] v_{2}\right\}}
\end{aligned}
$$

In general, $Q\left(F_{0}, p_{0}\right)$ has only one or one pair of eigenvalues on the imaginary axis. Suppose the pair is $\lambda_{1,2}\left[Q\left(F_{0}, p_{0}\right)\right]= \pm j \omega$, then $\rho_{M}(F)$ is continuously differentiable at $F_{0}$ if $\frac{\partial \operatorname{Re} \lambda_{1}\left[Q\left(F_{0}, p_{0}\right)\right]}{\partial p} \neq 0$ since $\frac{\partial \rho_{M}^{1}(F)}{\partial F}$ and $\frac{\partial \rho_{M}^{2}(F)}{\partial F}$ are equal.

By Theorem 1, a gradient based algorithm can be devised to increase $\rho_{M}(F)$. The constraint that $A_{0}+B_{0} F$ is stable will be guaranteed in each step since $\rho_{M}(F)$ is increased after each iteration. In the following section, we will present a method to increase $\rho_{M}(F)$ under the pole assignment constraint.

\section{Optimizing Stability Margin Under Pole Assignment}

Let $\left\{\lambda_{1}, \lambda_{2}, \cdots, \lambda_{n}\right\}$ be a set of self-conjugate complex numbers corresponding to the set of desired poles. Assume that there are $n^{\prime}$ complex conjugate pairs, $\lambda_{2 i-1}, \lambda_{2 i}=\alpha_{i} \pm j \beta_{i}, i=1,2, \ldots, n^{\prime}$, then one can define the following real block diagonal matrix:

$\Lambda:=\operatorname{diag}\left[\left[\begin{array}{cc}\alpha_{1} & \beta_{1} \\ -\beta_{1} & \alpha_{1}\end{array}\right], \ldots,\left[\begin{array}{cc}\alpha_{n^{\prime}} & \beta_{n^{\prime}} \\ -\beta_{n^{\prime}} & \alpha_{n^{\prime}}\end{array}\right], \lambda_{2 n^{\prime}+1}, \ldots, \lambda_{n}\right]$

It is assumed that the eigenvalues of $\Lambda$ are distinct, then for a given controllable pair $(A, B), A \in \mathbf{R}^{n \times n}$ and $B \in \mathbf{R}^{n \times m}$, the problem of pole assignment by state feedback is to choose feedback matrix $F$, such that

$$
V^{-1}(A+B F) V=\Lambda
$$

for some nonsingular $V$.

Now we turn back to system (3). At the nominal working point $p=0$, the closed-loop state matrix is $A_{0}+B_{0} F$. It is required that the eigenvalues of $A_{0}+B_{0} F$ be the set $\left\{\lambda_{1}, \lambda_{2}, \cdots, \lambda_{n}\right\}$. Assume that $\left(A_{0}, B_{0}\right)$ is controllable and $\operatorname{rank}\left(B_{0}\right)>1$, then there are infinity many $F$ that satisfy this pole assignment constraint. Our objective is to choose among these $F$ such that the stability margin $\rho_{M}(F)$ is maximized. This problem can be formulated as:

$$
\sup \rho_{M}(F) \quad \text { s.t. } \quad V^{-1}\left(A_{0}+B_{0} F\right) V=\Lambda
$$

In the following, we will follow the idea of $[1,2]$ to parametrize all the feedback matrices $F$ that satisfy (11) as the function of a free parameter $U \in \mathbf{R}^{m \times n}$. This is achieved by solving a parametric Sylvester 
equation in $U$ and then recovering the feedback matrix $F=U V^{-1}$. In this way, $\rho_{M}(F)$ becomes a function of the free parameter $U$. Explicit formulas to compute the gradient can be derived.

Given a controllable pair $\left(A_{0}, B_{0}\right)$ and a real block diagonal matrix $\Lambda$ with the form in (10) such that $A_{0}$ and $\Lambda$ have no common eigenvalues, then a function $f: U \rightarrow F$ is defined as follows. For $U \in \mathbf{R}^{m \times n}$, solve

$$
A_{0} V-V \Lambda=-B_{0} U
$$

for $V$ and if $V$ is nonsingular, let $F=U V^{-1}$. The function is denoted as $F=f(U)$. The domain of $f$ is

$$
\mathcal{D}_{f}:=\left\{U \in \mathbf{R}^{m \times n} \mid V \text { in (13) is nonsingular }\right\}
$$

and the range of $f$ is $\mathcal{R}_{f}=f\left(\mathcal{D}_{f}\right)$. It is easy to see that $V$ depends linearly on $U$ and $F$ is in fact a rational function of the elements of $U$.

Theorem $2[1,4]$

(a) $\mathcal{D}_{f}$ is a dense open set in $\mathbf{R}^{m \times n}$.

(b) $\left\{F: V^{-1}\left(A_{0}+B_{0} F\right) V=\Lambda\right\}=\mathcal{R}_{f}=f\left(\mathcal{D}_{f}\right)$.

The above result shows that $F$ satisfying the constraint in (12) can be parametrised as a function of $U$ and justifies its use as an optimization parameter. Since $\rho_{M}(F)$ is a function of $F$ which is in turn uniquely determined by $U$, consequently, it can be expressed as $J(U):=\rho_{M}(F(U))$. By the above Theorem, the constraint in (12) can be relaxed and we get an equivalent optimization problem

$$
\sup _{U \in \mathcal{D}_{f}} J(U)
$$

\subsection{Gradient Formula for Stability Margin}

As $F=f(U)$ is a rational function and $\mathcal{D}_{f}$ is an open set, so $F$ is differentiable with respect to $U$ for $U \in \mathcal{D}_{f}$. Thus $\frac{\partial J}{\partial U}$ exists if $\rho_{M}(F)$ is differentiable with respect to $F$.

Theorem 3 Suppose $U \in \mathcal{D}_{f}$ and

$$
A_{0} V-V \Lambda=-B_{0} U \quad, \quad F=U V^{-1}
$$

If $\frac{\partial \rho_{M}(F)}{\partial F}$ exists, then

$$
\frac{\partial J}{\partial U} \equiv \frac{\partial \rho_{M}(F(U))}{\partial U}=\left(\frac{\partial \rho_{M}}{\partial F}\right) V^{-T}+B_{0}^{T} Y^{T}
$$

where $V^{-T}$ denotes $\left(V^{-1}\right)^{T}$ and $Y$ is the unique solution of

$$
Y A_{0}-\Lambda Y=V^{-1}\left(\frac{\partial \rho_{M}}{\partial F}\right)^{T} F
$$

Proof: Omitted.

\subsection{Schematic Numerical Algorithm}

1. STEP 1: Select $U_{0} \in \mathcal{D}_{f}$ and a small $\epsilon>0$.

2. STEP 2: Compute $J\left(U_{0}\right)$.

(a) Solve $A_{0} V-V \Lambda=-B_{0} U_{0}$ for $V$, let $F_{0}=$ $U_{0} V^{-1}$.

(b) Compute $\rho_{M}\left(F_{0}\right)$ by the bisection method.

If Condition 2 of Theorem 1 is not satisfied, then stop the algorithm.

3. STEP 3: Compute $\frac{\partial J}{\partial U}$.

(a) Compute $F_{0}=f\left(U_{0}\right)$ and $p_{0}=J\left(U_{0}\right)=$ $\rho_{M}\left(F_{0}\right)$.

(b) Compute $\frac{\partial \rho_{M}}{\partial F}$ via (9) (provided that Condition 2 in Theorem 1 is satisfied).

(c) compute $\frac{\partial J}{\partial U}$ via (15) and (16).

4. STEP 4: Maximize $J\left(U_{0}+h \frac{\partial J}{\partial U}\right)$ with respect to $h$ (line search).

5. STEP 5: If $J\left(U_{0}+h \frac{\partial J}{\partial U}\right)-J\left(U_{0}\right)<\epsilon$, then stop. Otherwise replace $U_{0}$ with $U_{0}+h \frac{\partial J}{\partial U}$ and go to STEP 2.

\section{Numerical Examples}

Consider the system of two identical penduli coupled by a spring [9],

$\dot{x}=\left[\begin{array}{cccc}0 & 1 & 0 & 0 \\ \frac{g}{l}-\frac{k \alpha^{2}}{m l^{2}} & 0 & \frac{k \alpha^{2}}{m l^{2}} & 0 \\ 0 & 0 & 0 & 1 \\ \frac{k \alpha^{2}}{m l^{2}} & 0 & \frac{g}{l}-\frac{k \alpha^{2}}{m l^{2}} & 0\end{array}\right] x+\left[\begin{array}{cc}0 & 0 \\ \frac{1}{m l^{2}} & 0 \\ 0 & 0 \\ 0 & \frac{1}{m l^{2}}\end{array}\right] u$

Example 1: Suppose $\alpha$ is the uncertain parameter and other parameters are constants: $l=1, k=$ $2, m=0.2$. The nominal value of $\alpha$ is $\sqrt{0.5}$. Let $p=\alpha^{2}-0.5$, then

$$
A(p)=A_{0}+p A_{1}, \quad B(p)=B_{0}
$$

where

$$
\begin{aligned}
A_{0}= & {\left[\begin{array}{cccc}
0 & 1 & 0 & 0 \\
4.8 & 0 & 5 & 0 \\
0 & 0 & 0 & 1 \\
5 & 0 & 4.8 & 0
\end{array}\right], B_{0}=\left[\begin{array}{ll}
0 & 0 \\
5 & 0 \\
0 & 0 \\
0 & 5
\end{array}\right] } \\
A_{1} & =\left[\begin{array}{cccc}
0 & 1 & 0 & 0 \\
-10 & 0 & 10 & 0 \\
0 & 0 & 0 & 1 \\
10 & 0 & -10 & 0
\end{array}\right]
\end{aligned}
$$

The characteristic equation of $A(p)=A_{0}+p A_{1}$ is

$$
\left[5 s^{2}-49(p+1)\right]\left[5 s^{2}+(100 p+1)(p+1)\right]=0
$$


Thus, the open-loop system is always unstable and the nominal system matrix $A_{0}$ has eigenvalues \pm 3.1305 and $\pm j 0.4472$. The desired closed-loop eigenvalues of $A_{0}+B_{0} F$ are $,-1 \pm j,-2,-3$.

Let $U_{0}=\left[\begin{array}{cccc}0 & 1 & -1 & 0 \\ 1 & 0 & 0 & -1\end{array}\right]$, we obtain the initial feedback matrix $F_{0}$ with stability margin given by $J\left(U_{0}\right)=\rho_{M}\left(F\left(U_{0}\right)\right)=0.0844$. After 4 iterations, the gradient algorithm terminates at $F_{4}$ given by

$$
F_{4}=\left[\begin{array}{cccc}
-2.0849 & -0.9109 & -1.6376 & -0.2889 \\
-01118 & 0.2245 & -0.8832 & -0.4891
\end{array}\right]
$$

and $\rho_{M}\left(F_{4}\right)=0.3004$ which represents a significant improvement of the size of the stability margin. In fact, when $p=-0.3004$, the system has a pair of poles coalesce at the origin that destabilize the system.

Example 2: Now suppose $l$ is the uncertain parameter. The other parameters are constants, $k=2, \alpha=$ $\sqrt{0.5}, m=0.2$. The nominal value of $l$ is 1 . Let $p=\frac{1}{l}-1$, then

$A(p)=A_{0}+p A_{1}+p^{2} A_{2}, \quad B(p)=B_{0}+p B_{1}+p^{2} B_{2}$

where $A_{0}, B_{0}$ are the same as those in Example 1 and

$A_{1}=\left[\begin{array}{rrrr}0 & 0 & 0 & 0 \\ -0.2 & 0 & 10 & 0 \\ 0 & 0 & 0 & 0 \\ 10 & 0 & -0.2 & 0\end{array}\right], B_{1}=\left[\begin{array}{rr}0 & 0 \\ 10 & 0 \\ 0 & 0 \\ 0 & 10\end{array}\right]$
$A_{2}=\left[\begin{array}{rrrr}0 & 0 & 0 & 0 \\ -5 & 0 & 5 & 0 \\ 0 & 0 & 0 & 0 \\ 5 & 0 & -5 & 0\end{array}\right], B_{2}=\left[\begin{array}{ll}0 & 0 \\ 5 & 0 \\ 0 & 0 \\ 0 & 5\end{array}\right]$

The characteristic equation is

$\left[5 s^{2}-49(p+1)\right]\left[5 s^{2}+(p+1)\left(50 p^{2}+100 p+1\right)\right]=0$

Similarly, the system is always unstable without feedback. The desired closed loop eigenvalues are the same as Example 1.

Different local minima with the same value of $\rho_{M}(F)$ are detected. The optimal stability magrin is $\rho_{M}\left(F^{*}\right)=$ 0.2428. A particular optimal feedback that achieve this stability margin is $F^{*}$ given below

$$
F^{*}=\left[\begin{array}{llll}
-1.2510 & -0.5367 & -0.7251 & -0.0740 \\
-1.5804 & -0.2938 & -2.0610 & -0.8633
\end{array}\right]
$$

\section{References}

[1] Bhattacharyya, S.P. and de Souza, E., 1982, Pole assignment via Sylvester equation, Systems 8 Control Letters, 1, pp. 261-263.
[2] Cavin III, R. K. and Bhattacharyya, S. P., 1983, Robust and well conditioned eigenstructure assignment via Sylvester's equation, Optimal Control: Applic. and Methods, 4, pp. 205-212.

[3] Hinrichsen, D. and Pritchard, A.J., 1990, Real and complex stability radii: a survey. In Control of Uncertain Systems, D. Hinrichsen and B. Mårtensson, Eds. Birkhäuser, pp. 119-162.

[4] Hu, T., and Shi, S., 1989, The set of feedback matrices that assign the poles of a system, Proc. of MTNS-89, 2, pp. 129-135.

[5] Kantor, J. C. and Spencer Jr, B. F., 1993, On real parameter stability margins and their computation, Int. J. Control, 57(2), pp. 453-462.

[6] Lam, J. and Yan, W., 1995, A gradient flow approach to robust pole-placement problem, Int. J. Robust and Nonlinear Control, 5, pp. 175-185.

[7] Patel, R.V. and Toda, M., 1980, Quantitative measures of robustness for multivariable systems, in Proc. Joint Automatic Control Conference, San Francisco, TP8-A.

[8] Sideris, A. and Sánchez Peña, 1989, Fast computation of the multivariable stability margin for real interrelated uncertain parameters, IEEE Transactions on Automatic Control, 34(12), pp. 1272-1276.

[9] Siljak, D.D., 1989, Parameter space methods for robust control design: a guided tour, IEEE Transactions on Automatic Control, 34(7), pp. 674-688.

[10] Tesi, A. and Vicino, A., 1989, A new fast algorithm for robust stability analysis of control systems with linearly dependent parametric uncertainties, Systems \& Control Letters, 13, pp. 321-329.

[11] Tesi, A. and Vicino, A., 1990, Robust stability of state-space models with structured uncertainties, IEEE Transactions on Automatic Control, 35(2), pp. 191-195.

[12] Vicino, A., Tesi, A. and Milanese, M., 1990, Computation of nonconservative stability perturbation bounds for systems with nonlinearly correlated uncertainties, IEEE Transactions on Automatic Control, 35(7), pp. 835-841.

[13] Yedavalli, R.K., 1985, Perturbation bounds for robust stability in linear state space models, Int. J. Control, 42, pp. 1507-1517.

[14] Zhou, K. and Khargonekar, P.P., 1987, Stability robustness bounds for linear state-space models with structured uncertainty, IEEE Transactions on Automatic Control, 32, pp. 621-623. 